\title{
Preoperative brain computed tomographic perfusion for quantitative evaluation of cerebral malperfusion caused by acute type A aortic dissection
}

Yosuke Inoue, $\mathrm{MD},{ }^{\mathrm{a}}$ Manabu Inoue, $\mathrm{MD}, \mathrm{PhD},{ }^{\mathrm{b}}$ Masatoshi Koga, $\mathrm{MD}, \mathrm{PhD},{ }^{\mathrm{b}}$ and Hitoshi Matsuda, MD, PhD, ${ }^{a}$ Suita/Osaka, Japan

From the Departments of ${ }^{\mathrm{a} C}$ ardiovascular Surgery, and ${ }^{\mathrm{b}}$ Cerebrovascular Medicine, National Cerebral and Cardiovascular Center, Suita/Osaka, Japan.

Disclosures: The authors reported no conflicts of interest.

The Journal policy requires editors and reviewers to disclose conflicts of interest and to decline handling or reviewing manuscripts for which they may have a conflict of interest. The editors and reviewers of this article have no conflicts of interest.

Received for publication July 9, 2021; accepted for publication Sept 14, 2021; available ahead of print Sept 22, 2021.

Address for reprints: Hitoshi Matsuda, MD, PhD, Department of Cardiovascular Surgery, National Cerebral and Cardiovascular Center, 6-1 Kishibe-shimmachi, Suita, Osaka, 564-8565, Japan (E-mail: hitmat@mist.ocn.ne. jp).

JTCVS Techniques 2021;10:190-5

2666-2507

Copyright (C 2021 The Author(s). Published by Elsevier Inc. on behalf of The American Association for Thoracic

Surgery. This is an open access article under the CC BY-NC-ND license (http://creativecommons.org/licenses/bync-nd/4.0/).

https://doi.org/10.1016/j.xjtc.2021.09.031

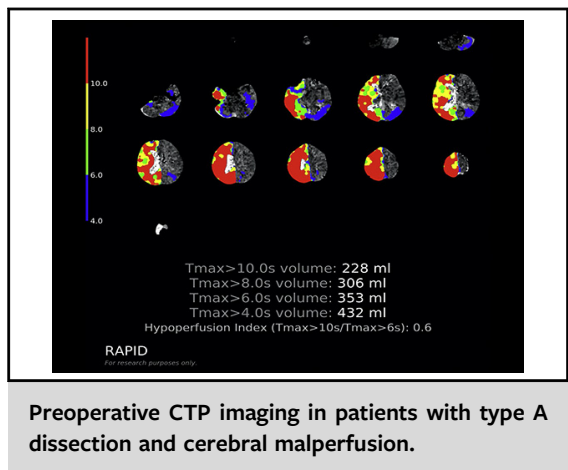

CENTRAL MESSAGE

Quantitative evaluation using CT perfusion is valuable in critical decision making, even in patients with cerebral malperfusion caused by acute type A aortic dissection.

See Commentaries on pages 196 and 198.
It is considerably challenging to make preoperative decisions for cerebral malperfusion caused by acute type A aortic dissection (AAAD) based on quantitative evaluation. Brain imaging with multimodal computed tomography (CT) has great potential to provide pathophysiologic information on acute ischemic stroke (AIS) that may be relevant to treatment decisions. Computed tomography perfusion (CTP) of the brain has been recommended by the American Heart Association/ American Stroke Association guidelines to assess patients eligible for thrombectomy by detecting the ischemic core and penumbra. ${ }^{1}$ We report 2 patients who underwent emergent central repair for AAAD after CTP, automatically assessed by software (RApid processing of Perfusion and Diffusion [RAPID]; IschemaView, Menlo Park, Calif) for the quantitative evaluation of cerebral malperfusion.

This study was approved by the institutional review board (M30-057/September 9, 2018). Individual written informed consents for the publication of their study data were obtained, and all authors have no conflict of interest in this study.

\section{CLINICAL SUMMARY Patient 1}

A 68-year-old female patient with a history of thoracic endovascular repair for descending aortic aneurysm presented with dysarthria followed by loss of consciousness. She was transferred from a community hospital 2 hours after the onset after a definitive diagnosis of AAAD with bilateral common carotid artery (CCA) dissection (Figure 1, A). She had fallen into a deep coma with stable hemodynamics, and CTP revealed massive penumbra $(735 \mathrm{~mL})$ without a complete ischemic core (Figure 1, B). Cardiopulmonary bypass was established through the right distal axillary artery for cerebral perfusion (Video 1) and femoral artery 3.5 hours after the onset of AAAD. Total arch replacement with frozen elephant trunk was performed subsequently. The amount of penumbra dramatically improved $(10 \mathrm{~mL})$ immediately after surgery (Figure 1,C). The patient recovered consciousness and linguistic ability, with only right-arm monoparesis reported. 


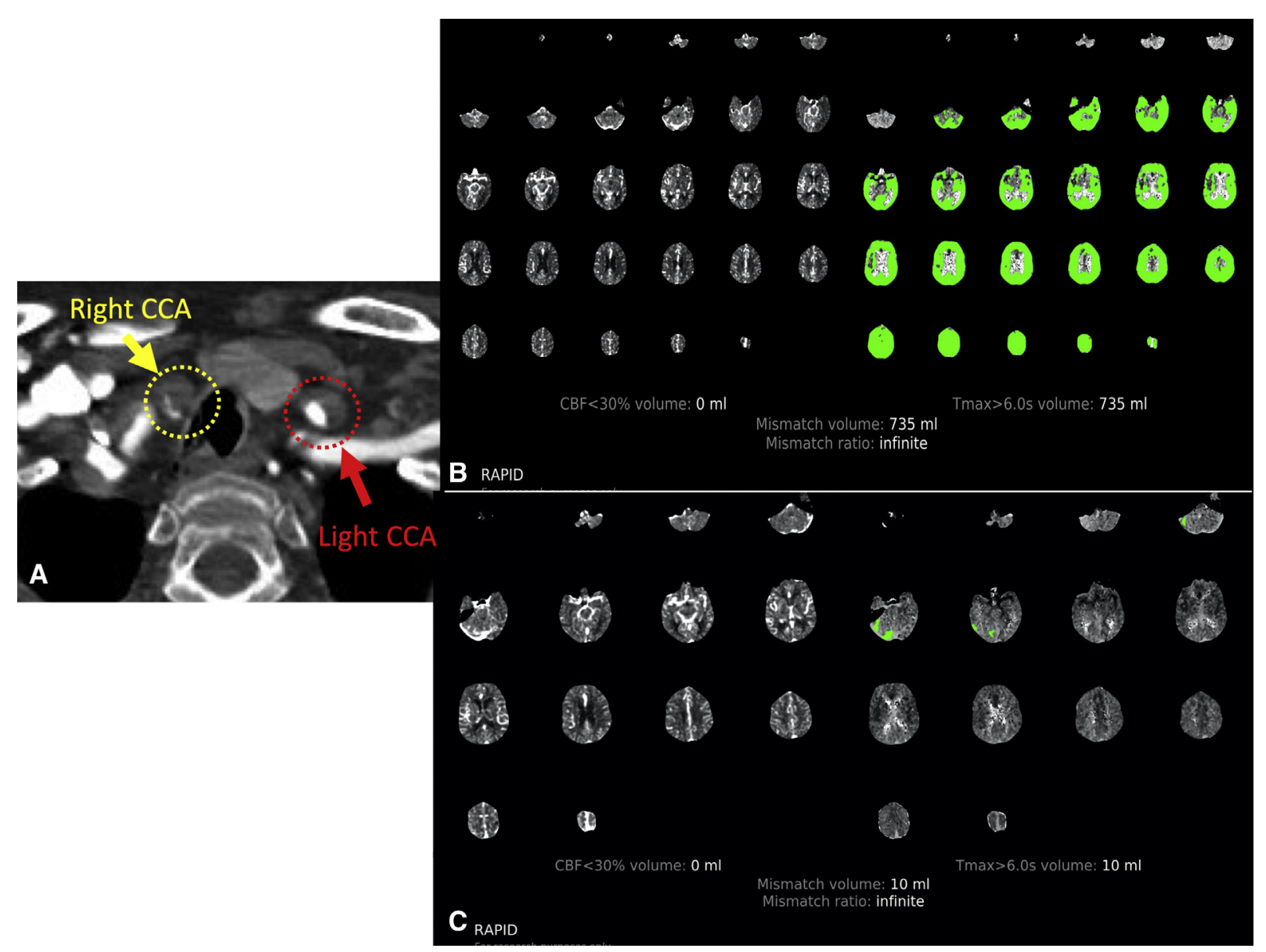

FIGURE 1. Illustration summarizing case 1. A, Preoperative CT angiography focusing on CCA stenosis. Yellow circle points out right CCA with decompression of true lumen. Red circle points out left CCA with decompression of true lumen. B, Preoperative CT perfusion imaging automated by software (RAPID). Left side implies no ischemic core, and green lesion (right side) implies occupied area of penumbra. C, Postoperative CT perfusion imaging automated by software (RAPID). Left side implies no ischemic core and green penumbra lesion (right side) dramatically decreased. CCA, Common carotid artery; $C B F$, cerebral blood flow; RAPID, RApid processing of Perfusion and Diffusion.

\section{Patient 2}

A 67-year-old female patient who was found to have collapsed and was transferred with left hemiparesis

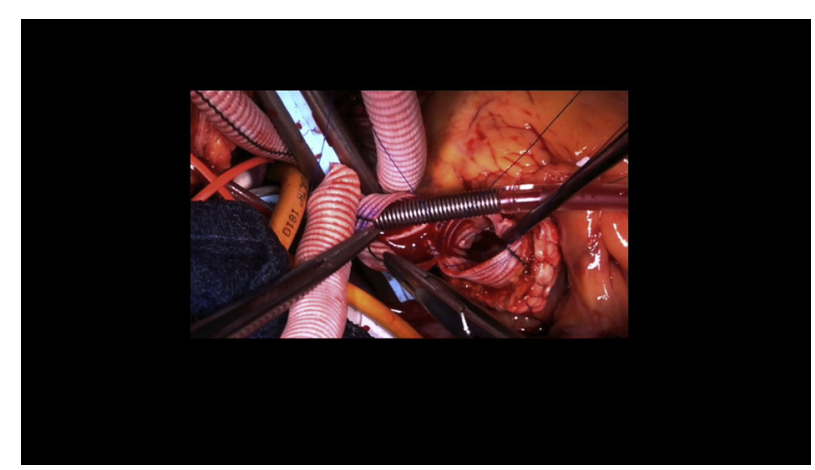

VIDEO 1. Explanation of CTP data and surgical strategy for patients with acute type A aortic dissection. Video available at: https://www.jtcvs.org/ article/S2666-2507(21)00662-3/fulltext.
2 hours after last known well. She was hemodynamically stable, Glasgow Coma Scale score was 11 (E3, V4, and M4), and CT revealed AAAD with static obstruction of the right CCA. CTP was also checked immediately after whole-body CT angiography (Figure 2, A), which revealed ischemic core $(57 \mathrm{~mL})$ and a high volume $(228 \mathrm{~mL})$ of Tmax $>10$ seconds, which meant the brain area perfused more than 10 seconds with poor brain collaterals and that the hypoperfusion intensity ratio (Tmax $>10$ seconds/Tmax $>6$ seconds $=0.6)$ increased, which meant the brain ischemia was progressing fast (Figure 2, B). Emergency total arch replacement with frozen elephant trunk, which revascularized right CCA by the individual supra-aortic branch reconstruction, was started 2.5 hours after hospital arrival. Massive intracranial bleeding was ultimately diagnosed postoperatively (Figure 2, C), and her neurologic status was deteriorated to a vegetative despite the effort with craniotomy. 


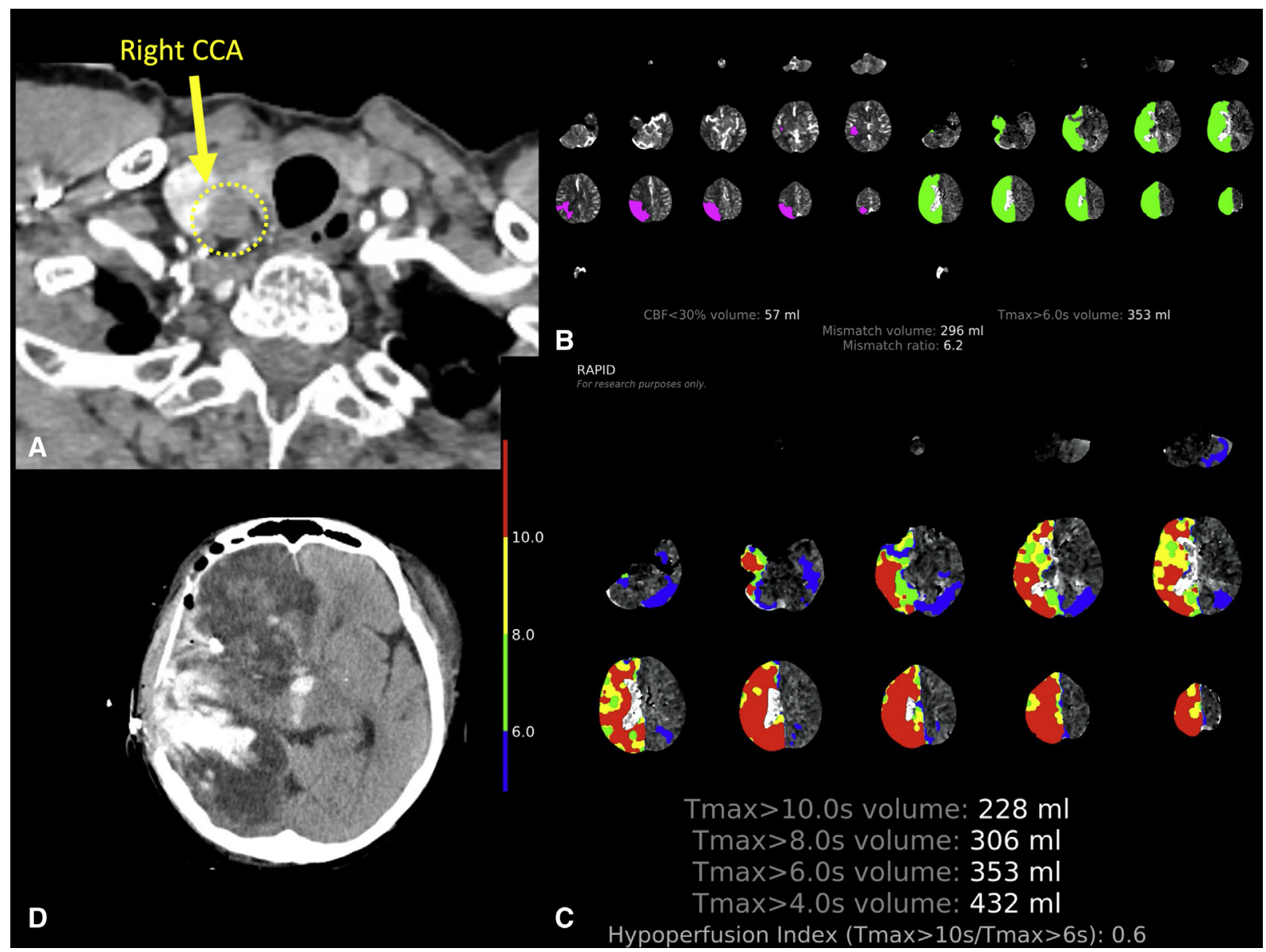

FIGURE 2. Illustration summarizing case 2. A, Preoperative CT angiography focusing on CCA stenosis. Yellow circle points out right CCA with static obstruction. B, Preoperative CT perfusion imaging automated by software (RAPID). Left side implies ischemic core was $57 \mathrm{~mL}$ and green lesion (right side) implies occupied area of penumbra as much as $353 \mathrm{~mL}$. C, Preoperative CT perfusion imaging automated by software (RAPID). Tmax $>10$ seconds volume was detected as $228 \mathrm{~mL}$ with greater hypoperfusion intensity ratio as 0.6 . D, Postoperative CT of the head revealed massive intracranial hemorrhage requiring craniotomy. $C C A$, Common carotid artery; $C B F$, cerebral blood flow; RAPID, RApid processing of Perfusion and Diffusion.

\section{DISCUSSION}

Cerebral malperfusion with AAAD has been frequently discussed based on preoperative symptoms, which can be fluctuating, subjective, and influenced by shock or cardiac arrest. $^{2,3}$ A consistent surgical strategy based on objective evaluation is thus critical.

Intracranial CT angiography has been attempted to evaluate the severity of malperfusion. ${ }^{4}$ The objective of angiography is to reveal a lack or delay in perfusion but is qualitative to evaluate cerebral blood flow. For quantitative evaluation, we applied CTP, which has been approved by guidelines to determine the treatment strategy for AIS. ${ }^{1}$ CTP imaging was performed on multidetector helical CT scanners in cine mode. Perfusion parameters (regional cerebral blood flow and Tmax) were automatically computed with RAPID software using a delayindependent deconvolution approach. Based on recent data suggesting cerebral blood flow is more accurate than cerebral blood volume for defining ischemic core, irreversible "ischemic core" was prespecified as regional cerebral blood flow $<30 \%$ of contralateral hemisphere median. Contrary, salvageable "penumbra" was prespecified as spital area Tmax $\geq 6$ seconds. According to these parameters, ischemic core and penumbra mismatch map was visualized to determine the treatment strategy for the brain. [Sup ref] Figure E1 demonstrates the example of mismatch map.

The effectiveness of evaluating ongoing cerebral ischemia evoked by cerebral malperfusion due to AAAD by CTP has not been established. In patient 1 , who presented a massive penumbra without an ischemic core, emergency central repair to restore cerebral perfusion was justified regardless of the preoperative symptoms. In patient 2 , despite the moderate severity of symptom 2 hours after the onset, a large volume of Tmax $>10$ seconds with increased hypoperfusion intensity ratio indicating an 
insufficient blood supply from the collateral artery had suggested no necessity or contraindication of restoration of cerebral perfusion. Olivot and colleagues ${ }^{5}$ suggested a hypoperfusion intensity ratio $>0.5$ could predict infarct growth and poor clinical outcomes. Generally, in patients with AIS, regional cerebral blood flow $<30 \%$ greater than $53 \mathrm{~mL}$ and/or Tmax delay of $>10$ seconds greater than $100 \mathrm{~mL}$ have been reported as the prognosis of malignant profile [Sup ref]. According to hypoperfusion intensity ratio and aforementioned criteria, patient 2 would be included in the malignant profile.

Another option ligating the culprit carotid artery with total arch replacement to minimize the burden of reperfusion should be considered. The contrasting 2 patients presented quite similar preoperative presentations, including age, sex, and duration from the onset to hospital arrival except for CTP results. Further investigation must be warranted for new methodologies. However, quantitative evaluation using CTP is suggested to be valuable in critical decision-making, even in patients with cerebral malperfusion due to AAAD.

\section{References}

1. Powers WJ, Rabinstein AA, Ackerson T, Adeoye OM, Bambakidis NC, Becker K, et al. 2018 guidelines for the early management of patients with acute ischemic stroke: a guideline for healthcare professionals from the American Heart Association/American Stroke Association. Stroke. 2018;49:e46-110.

2. Sultan I, Bianco V, Patel HJ, Arnaoutakis GJ, Eusanio MD, Chen EP, et al. Surgery for type A aortic dissection in patients with cerebral malperfusion: results from the International Registry of Acute Aortic Dissection. J Thorac Cardiovasc Surg. 2021;161:1713-20.

3. Pocar M, Passolunghi D, Moneta A, Mattioli R, Donatelli F. Coma might not preclude emergency operation in acute aortic dissection. Ann Thorac Surg. 2006;81:1348-51.

4. Fukuhara S, Norton EL, Chaudhary N, Burris N, Shiomi S, Kim KM, et al. Type A aortic dissection with cerebral malperfusion: new insights. Ann Thorac Surg. 2021;112:501-9.

5. Olivot JM, Mlynash M, Inoue M, Marks MP, Wheeler HM, Kemp S, et al Hypoperfusion intensity ratio predict infarct progression and functional outcome in the DEFUSE 2 cohort. Stroke. 2014;45:1018-23. 


\section{E-References}

E1. Campbell BC, Weir L, Desmond PM, Tu HT, Hand PJ, Yan B, et al. CT perfusion improves diagnostic accuracy and confidence in acute ischemic stroke. J Neurol Neurosurg Psychiatry. 2013;84:613-8.

E2. Inoue M, Mlynash M, Straka M, Lansberg MG, Zaharchuk G, Bammer R, et al. Patients with the malignant profile within 3 hours of symptom onset have very poor outcomes after intravenous tissue-type plasminogen activator therapy. Stroke. 2012;43:2494-6.
E3. Mokin M, Levy El, Saver JL, Siddiqui AH, Goyal M, Bonafe A, et al. Predictive value of RAPID assessed perfusion thresholds on final infarct volume in SWIFT PRIME (solitaire with intension for thrombectomy as primary endovascular treatment). Stroke. 2017;48:932-8.

E4. Ganesh A, Goyal M. Thrombectomy for acute ischemic stroke: recent insights and future directions. Curr Neurol Neurosci Rep. 2018; 18:59. 


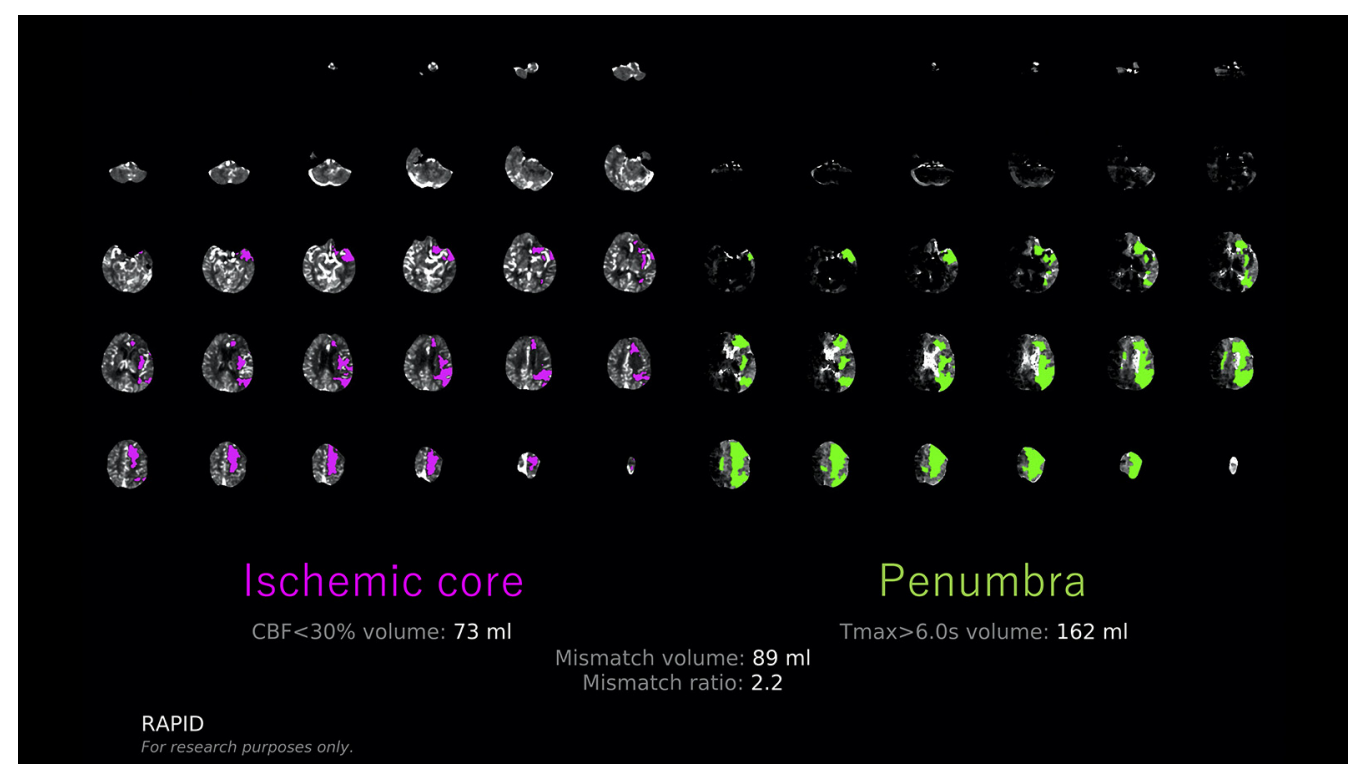

FIGURE E1. An example of ischemic core penumbra mismatch map automatically computed by RAPID software. CBF, Cerebral blood flow; $R A P I D$, RApid processing of Perfusion and Diffusion. 